\title{
Electron beam energy chirp control with a rectangular corrugated structure at the Linac Coherent Light Source
}

\author{
Zhen Zhang, ${ }^{1,2}$ Karl Bane, ${ }^{1}$ Yuantao Ding, ${ }^{1}$ Zhirong Huang, ${ }^{1}$ Richard Iverson, ${ }^{1}$ \\ Timothy Maxwell, ${ }^{1}$ Gennady Stupakov, ${ }^{1}$ and Lanfa Wang ${ }^{1}$ \\ ${ }^{1}$ SLAC National Accelerator Laboratory, Menlo Park, California 94025, USA \\ ${ }^{2}$ Department of Engineering Physics, Tsinghua University, Beijing 100084, China
}

(Received 17 October 2014; published 30 January 2015)

\begin{abstract}
Electron beam energy chirp is an important parameter that affects the bandwidth and performance of a linac-based, free-electron laser. In this paper we study the wakefields generated by a beam passing between flat metallic plates with small corrugations, and then apply such a device as a passive dechirper for the Linac Coherent Light Source (LCLS) energy chirp control with a multi-GeV and femtosecond electron beam. Similar devices have been tested in several places at relatively low energies $(\sim 100 \mathrm{MeV})$ and with relatively long bunches ( $>1 \mathrm{ps}$ ). In the parameter regime of the LCLS dechirper, with the corrugation size similar to the gap between the plates, the analytical solutions of the wakefields are no longer applicable, and we resort to a field matching program to obtain the wakes. Based on the numerical calculations, we fit the short-range, longitudinal wakes to simple formulas, valid over a large, useful parameter range. Finally, since the transverse wakefields - both dipole and quadrupole-are strong, we compute and include them in beam dynamics simulations to investigate the error tolerances when this device is introduced in the LCLS.
\end{abstract}

DOI: 10.1103/PhysRevSTAB.18.010702

PACS numbers: $41.60 . \mathrm{Cr}$

\section{INTRODUCTION}

In a linac-based X-ray free electron laser (FEL) there is often a need for energy chirp control of the beam as the magnetic compression employed in such FELs [1-4] typically leaves an undesired time-energy correlation in the bunch. Such a chirp, if left uncorrected, can broaden the FEL bandwidth and degrade FEL performance $[5,6]$. While the chirp can be removed by off-crest acceleration in a following linac section, this solution can be costly or impractical, particularly for a superconducting linac-based FEL. For such cases, a dedicated passive structure that can intentionally generate a strong longitudinal wakefield was recently proposed to "dechirp" the beam. Additionally, the dechirper can do the opposite to make X-ray pulses with a broader energy spectrum and generate ultrashort pulses for a variety of applications.

In Ref. [7], a round metallic structure with corrugated walls was suggested and analyzed as a passive dechirper. Compared to the round geometry, the flat geometry using two corrugated plates has the advantage of allowing the dechirper strength to be adjusted by changing the separation of the plates [8]. In both round and flat structures, the short-range transverse wakes can be strong, with amplitude scaling as the -4 th power of aperture (versus the -2 nd

Published by the American Physical Society under the terms of the Creative Commons Attribution 3.0 License. Further distribution of this work must maintain attribution to the author(s) and the published article's title, journal citation, and DOI. power for the longitudinal wake). In a flat structure, however, in addition to the usual dipole wakefield that is excited when the beam passes through off axis, there is also a quadrupole wake excited, even when the beam moves exactly along the symmetry axis. These transverse wakes will, if not properly controlled, increase the projected transverse emittance and lead to a deterioration in FEL performance.

Recently dechirper systems have been tested in Pohang, Brookhaven, and Shanghai at relatively low energies $(\sim 100 \mathrm{MeV})$ [9-11]. However, when this structure is used for multi-GeV beams, such as the several $\mathrm{GeV}$ beams of the Linac Coherent Light Source (LCLS) [12,13], in order to generate a significant dechirping effect within a reasonable length of structure, the gap between the two plates needs to be set very small (e.g., $\sim 1 \mathrm{~mm}$ ), which will introduce strong transverse effects. In addition, from the point of view of manufacturing feasibility with relaxed tolerances, the preferred size of corrugations should not be too small (e.g., $\gtrsim 0.5 \mathrm{~mm}$ ). In this case, the corrugation parameters become comparable to the gap of the two plates and the analytical solutions of wakefields for the structure [7,14] are no longer applicable.

In this paper we adopt the field matching method [15] to calculate the longitudinal and transverse wakefields of the flat corrugated structure that will serve as a dechirper for the LCLS. For the longitudinal case, we, in addition, perform wake calculations over a wide parameter range and find a simple fitting formula that can be useful for quickly finding the short-range wake of such devices. In these 


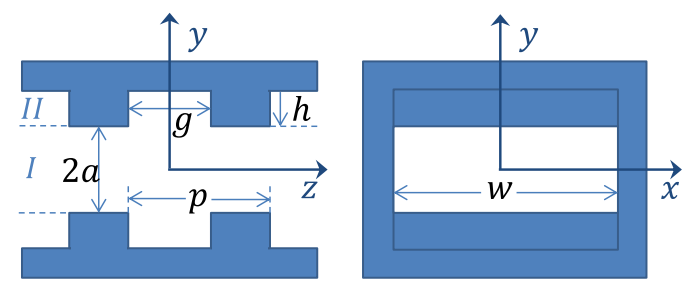

FIG. 1. Geometry of dechirper parameters: longitudinal cut with two periods (left) and transverse cut (right).

studies, it is found that higher band modes arise with small gap size. The amplitude of the rising second band modes is evaluated numerically for comparison. Finally, for the application to the LCLS, we study the effects of the transverse wakefields on beam quality and the tolerances these imply.

The structure we consider in this paper is a periodic, rectangular structure, two periods of which are sketched in Fig. 1. The parameters are: half-gap $a$ and width $w$; corrugation parameters: depth $h$, period $p$, opening $g$. The nominal parameters of the proposed LCLS dechirper are shown in Table I. They will be used in illustrative calculations of wakefields in the following sections. The appropriateness of this choice of parameters for LCLS applications will be addressed in Sec. III.

This paper is organized as follows. In Sec. II we introduce the field matching method and present some numerical results. Fitting formulas of the longitudinal wakefield are obtained based on the field matching calculations, which are given in Appendix A. The application of this structure to the LCLS of this structure is analyzed in Sec. III, including simulations of chirp control, the effects of the transverse wakefields and the subsequent tolerances for the beam position jitter and the misalignment of the structure necessary to preserve the transverse beam emittance. Finally, in Sec. IV we give concluding remarks.

\section{WAKEFIELDS AND FIELD MATCHING}

\section{A. Analytic formulas}

The analytical solutions of the wakefields for the structure in Fig. 1 are developed from the ones for a round pipe with similar corrugations $[16,17]$. For round geometry,

TABLE I. Structure parameters of LCLS dechirper.

\begin{tabular}{lcc}
\hline \hline Parameter & Value & Units \\
\hline Half-gap $a$ & $>0.5^{\mathrm{a}}$ & $\mathrm{mm}$ \\
Period $p$ & 0.5 & $\mathrm{~mm}$ \\
Depth $h$ & 0.5 & $\mathrm{~mm}$ \\
Opening $g$ & 0.25 & $\mathrm{~mm}$ \\
Width $w$ & 12 & $\mathrm{~mm}$ \\
Total length $L$ & $4^{\mathrm{b}}$ & $\mathrm{m}$ \\
\hline \hline
\end{tabular}

${ }^{\mathrm{a}}$ Nominal half-gap is $0.7 \mathrm{~mm}$.

${ }^{\mathrm{b}}$ The dechirper is composed of two sections of $2 \mathrm{~m}$ each. if we assume all corrugation dimensions are much smaller than the gap size ( $p, h \ll a$, with $a$ the radius) and the corrugations are relatively deep $(h \gtrsim p)$, then the point charge wake can be written as a pure cosine oscillation with one mode wave number $k=\sqrt{2 p /(a h g)}$ and with amplitude $Z_{0} c /\left(\pi a^{2}\right)\left(Z_{0}=377 \Omega\right.$ is the characteristic impedance of free space and $c$ is the speed of light). And the dipole wakefield is given by a sine oscillation of the same frequency, with the slope at the origin of $W_{d}^{\prime}\left(0^{+}\right)=2 Z_{0} c /\left(\pi a^{4}\right)$. There is no quadrupole wakefield.

For the flat geometry, the wakefield is more complicated than a simple cosine function. However, if we assume a large aspect ratio, $(w / 2 a)$, and only care about the wakefield over the short range, we can still approximate its longitudinal wake by a single frequency oscillation, with a modified amplitude factor: [15]

$$
W_{\|}(z)=\frac{\pi^{2}}{16} \frac{Z_{0} c}{\pi a^{2}} H(z) \cos (k z) 0<k z \lesssim 3 \pi,
$$

where $z$ is the distance the test particles is behind the driving particle and $H(z)=1(0)$ when $z>0(<0)$. For small corrugations, the wave number $k$ is well approximated by [15]

$$
k=\sqrt{\frac{p}{a h g}} .
$$

In flat geometry there are both dipole and quadrupole transverse wakes, where the total vertical wake effect near the $y=0$ symmetry plane is given by

$$
W_{y}=y_{0} W_{d}+y W_{q},
$$

with $y_{0}(y)$ the offset of the driving (test) particle. The shortrange transverse wakes are reasonably well approximated by sine functions with the same wave number $k$, and with slopes near the origin [18]

$$
W_{d}^{\prime}\left(0^{+}\right)=W_{q}^{\prime}\left(0^{+}\right)=\frac{2 Z_{0} c}{\pi a^{4}}\left(\frac{\pi}{4}\right)^{4} .
$$

Note that in Eqs. (1) and (4), the amplitudes of the wakefields are independent of the dimensions of corrugations. However, for structures with corrugation size comparable to $a$, such as the LCLS dechirper as shown in Table I, Eqs. (1) and (4) are no longer applicable and we need to use a numerical method, such as the field matching method, to find the wakefields.

\section{B. Field matching method}

The field matching method to solve the wakefields of the structure of Fig. 1 is described in an Appendix of Ref. [15]. We have written a Mathematica program based on this formalism with which we perform our parameter studies. 
We sketch the method briefly here; the reader is referred to Ref. [15] for more details (though she should be warned that there are a few typos in the equations there). It is useful to understand the method, in order to better make sense of the parameters studies. In addition, the calculation of the transverse wakes, which were not addressed in Ref. [15], are also sketched out here. (Note that all derivations in this section are in Gaussian units.)

Field matching is used to find the synchronous (to a speed-of-light particle), resonant modes of the structure. The domain of calculation is one period of the structure in Fig. 1, which extends longitudinally to $z= \pm p / 2$. The domain is divided into two regions: Region I, the "tube region," extends to $y= \pm a$; Region II, the "cavity region," for $z \leq \pm g / 2$, extends beyond $y= \pm a$ to $y= \pm(a+h)$. In the field matching program smooth walls at $x= \pm w / 2$ are assumed. (In the real structure there are no side walls. However, the side walls do not affect the short-range wake, provided the structure width is chosen so that $w / a \gg 1$.)

We are interested in the steady-state wakes excited by the beam and assume that the fields of a mode have a time dependence $e^{j k c t}$, where $k$ is the mode wave number and $t$ is time. For either region the electric and magnetic fields can be obtained from two Hertz vectors $\Pi_{m}, \Pi_{e}$, that represent $\mathrm{TM}$ and TE components:

$$
\begin{aligned}
\mathcal{E} & =\nabla \times \nabla \times \Pi_{e}-j k \nabla \times \Pi_{m}, \\
\mathcal{H} & =\nabla \times \nabla \times \Pi_{m}+j k \nabla \times \Pi_{e} .
\end{aligned}
$$

Since there is no boundary variation in the $x$ direction the Hertz vectors have only an $x$ component. To satisfy the boundary condition at $x= \pm w / 2$, the fields vary as cosines and sines of $k_{x} x$ where

$$
k_{x}=\frac{l \pi}{w},
$$

with $l$ an odd integer. The general solution of the wake involves a summation over all $l$.

Consider modes with a given horizontal mode number $l$. If the modes with $\mathcal{E}_{z} \neq 0$ on axis are chosen, the Hertz potentials for each region can be written as the sum of space harmonics as follows. For region I:

$$
\begin{aligned}
& \Pi_{m x}^{I}=\sum_{n=-\infty}^{\infty}\left[B_{n} \cosh \left(k_{y n}^{I} y\right)\right] \sin \left(k_{x} x\right) e^{-j \beta_{n} z} \\
& \Pi_{e x}^{I}=\sum_{n=-\infty}^{\infty}\left[C_{n} \sinh \left(k_{y n}^{I} y\right)\right] \cos \left(k_{x} x\right) e^{-j \beta_{n} z}
\end{aligned}
$$

with

$$
\beta_{n}=\beta_{0}+\frac{2 \pi n}{p}, \quad k_{y n}^{I}=\sqrt{\beta_{n}^{2}-k^{2}+k_{x}^{2}},
$$

For region II:

$$
\begin{aligned}
\Pi_{m x}^{I I}= & \sum_{s=1}^{\infty} E_{s} \sin \left[k_{y s}^{I I}(a+h-y)\right] \sin \left(k_{x} x\right) \\
& \times \sin \left[\alpha_{s}\left(z+\frac{g}{2}\right)\right], \\
\Pi_{e x}^{I I}= & \sum_{s=0}^{\infty} F_{s} \cos \left[k_{y s}^{I I}(a+h-y)\right] \cos \left(k_{x} x\right) \\
& \times \cos \left[\alpha_{s}\left(z+\frac{g}{2}\right)\right],
\end{aligned}
$$

with

$$
\alpha_{s}=\frac{\pi s}{g}, \quad k_{y s}^{I I}=\sqrt{k^{2}-\alpha_{s}^{2}-k_{x}^{2}}
$$

The $B_{n}, C_{n}, E_{s}, F_{s}$, are expansion coefficients. The arbitrary phase constant is set to $\beta_{0}=k$, so that the modes of the system are those excited by the beam (the synchronous modes).

Then the tangential components of the fields $\mathcal{E}_{z, x}, \mathcal{H}_{z, x}$ in the two regions are obtained from the Hertz vectors and are matched in the matching planes, at $y= \pm a$ :

$$
\begin{gathered}
\mathcal{E}_{z, x}^{I}= \begin{cases}\mathcal{E}_{z, x}^{I I} & |z|<g / 2, \\
0 & g / 2<|z|<p / 2,\end{cases} \\
\mathcal{H}_{z, x}^{I}=\mathcal{H}_{z, x}^{I I} \quad|z|<g / 2 .
\end{gathered}
$$

From the orthogonality of the expansion functions a system of equations is obtained that can be written as a real, symmetric, homogeneous matrix equation involving the expansion coefficients. The solution will give the fields to an arbitrary scale factor. For numerical calculation the infinite matrix is truncated to finite size. If $\mathcal{N}$ is the largest value of $n$-the space harmonics number in region I-that is kept, the system matrix equation will be of dimension $2(2 \mathcal{N}+1) \times 2(2 \mathcal{N}+1)$. (The parameter $s$ is also truncated, so that its maximum value $\mathcal{S} \sim \mathcal{N}$.) The wave numbers at which the determinant of the resulting matrix vanishes correspond to the modes excited in the structure.

The loss factor $\kappa$ of each mode (which is defined per unit length of structure) can be calculated from the field components:

$$
\kappa=\frac{\left|\mathcal{E}_{0}\right|^{2} p}{4 u\left(1-v_{g} / c\right)},
$$

with $\mathcal{E}_{0}$ the synchronous component of the longitudinal field ( $n=0$ represents the synchronous space harmonic), $u$ the stored energy per period, and $v_{g}$ is the group velocity of the mode. Note that the arbitrary constant in the numerator and denominator cancel. 
The longitudinal wakefield is given as the sum over $l$, of the modes, which can be written as

$$
W_{\|}(z)=2 H(z) \sum_{l} \kappa_{l} \cos \left(k_{l} z\right)
$$

where $k_{l}\left(\kappa_{l}\right)$ is the wave number (loss factor) of mode $l$. The quadrupole wakefield can be obtained as

$$
W_{q}(z)=2 H(z) \sum_{l} \kappa_{\perp l}^{(q)} \sin \left(k_{l} z\right),
$$

where $\kappa_{\perp l}^{(q)}=(l \pi / w)^{2}\left(\kappa_{l} / k_{l}\right)$ is the kick factor of the quadrupole mode with horizontal mode number $l$.

For the dipole modes, we need to rewrite the Hertz potentials Eq. (7) in terms of the functions that yield $\mathcal{E}_{z}=0$ on axis (viz. by exchanging the sinh and cosh functions) and repeat the procedure above to obtain the corresponding wave numbers. The dipole kick factors are obtained as:

$$
\kappa_{\perp l}^{(d)}=\frac{\left|\frac{d \mathcal{E}_{0}}{d y}\right|^{2} p}{4 u\left(1-v_{g} / c\right) k_{l}^{(d)}},
$$

where $k_{l}^{(d)}$ is the wave number of the dipole modes. Then the dipole wakefield becomes

$$
W_{d}(z)=2 H(z) \sum_{l} \kappa_{\perp l}^{(d)} \sin \left(k_{l}^{(d)} z\right)
$$

Finally, note that in the field matching we have assumed perfect conductivity in the walls. This approximation is good in that the resistance of metallic walls has but a small effect on the wake of these structures. Also we have assumed that the steady-state solution is a good approximation to the wake. The so-called catch-up distance, $z_{c u}=$ $a^{2} / 2 \sigma_{z}$ ( $\sigma_{z}$ is the rms bunch length), is the approximate distance over which the transient response becomes the steady-state response. For representative LCLS dechirper parameters, $a=1 \mathrm{~mm}, \sigma_{z}=25 \mu \mathrm{m}, z_{c u}=25 \mathrm{~mm}$, which shows that the distance to steady-state indeed is small compared to the total structure length $L=4 \mathrm{~m}$.

We have programmed the truncated field-matching equations - for both longitudinal and dipole modes-into Mathematica. For given horizontal mode number $l$, in the longitudinal case the program obtains the wave number $k_{l}$, loss factor $\kappa_{l}$, and quadrupole kick factor $\kappa_{\perp l}^{(q)}$; for the dipole modes it obtains the dipole wave number $k_{l}^{(d)}$ and dipole kick factor $\kappa_{\perp l}^{(d)}$. Then, by performing the sums Eqs. (14), (15), and (17), we obtain the wakes.

\section{Numerical results}

In this section we numerically address the questions of what matrix size should be chosen, how many horizontal modes are needed (in the wake sums), and how strong is the effect of higher band modes. In addition, we perform longitudinal wake calculations over a large useful parameter range, and then provide fitting formulas that can be used for generating the wake without needing to resort to numerical calculations. (Details of the fitting are given in Appendix A.) We focus here on the longitudinal wake calculations because we want to accurately know the wake effect on the beam chirp, whereas for the transverse wake effects, which we want to keep under control, accuracy is not as important.

\section{Convergence studies}

We begin by studying the question of what is a reasonable matrix size for the calculations. We use the nominal parameters of the LCLS dechirper in Table I, with $a=0.7 \mathrm{~mm}$. Note that $(w / 2 a)=8.6$, meaning that the side walls have little effect on the wakes. We calculate the first three horizontal modes $(l=1,3,5)$ for different values of $\mathcal{N}$. The wave numbers and loss factors versus $\mathcal{N}$ are shown in Fig. 2. It can be seen that the two wakefield parameters converge quickly as functions of $\mathcal{N}$, and even a small number suffices $(\mathcal{N}>3)$. A similar kind of convergence was found for other horizontal modes and for other dechirper parameters. Consequently, in the following sections of this report, we choose $\mathcal{N}=5$-which corresponds to a system matrix of size 22-for the field matching calculations.

Another question is, how many horizontal modes (with mode number $l$ ) are needed to obtain a good approximation to the wakefields? As an example, we again consider the LCLS dechirper parameters. Figure 3 presents the wave number, longitudinal loss factors, and transverse kick factors as functions of horizontal mode number $l$. Note that, in the plot, the units for loss factor are $\left[\mathrm{mm}^{-2}\right]$ and for kick factor $\left[\mathrm{mm}^{-3}\right]$. Also note that for given $l$, the longitudinal and quadrupole wakefields have the same wave number. We can observe that for these dechirper
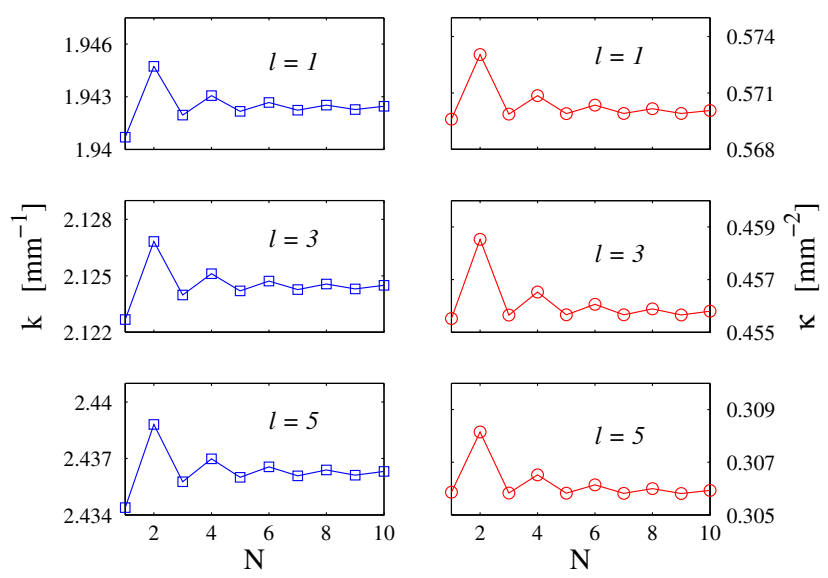

FIG. 2. Wave number (left) and loss factor (right) convergence versus $\mathcal{N}$ for $l=1,3,5$. The corresponding matrix size is $2(2 \mathcal{N}+1)$. 

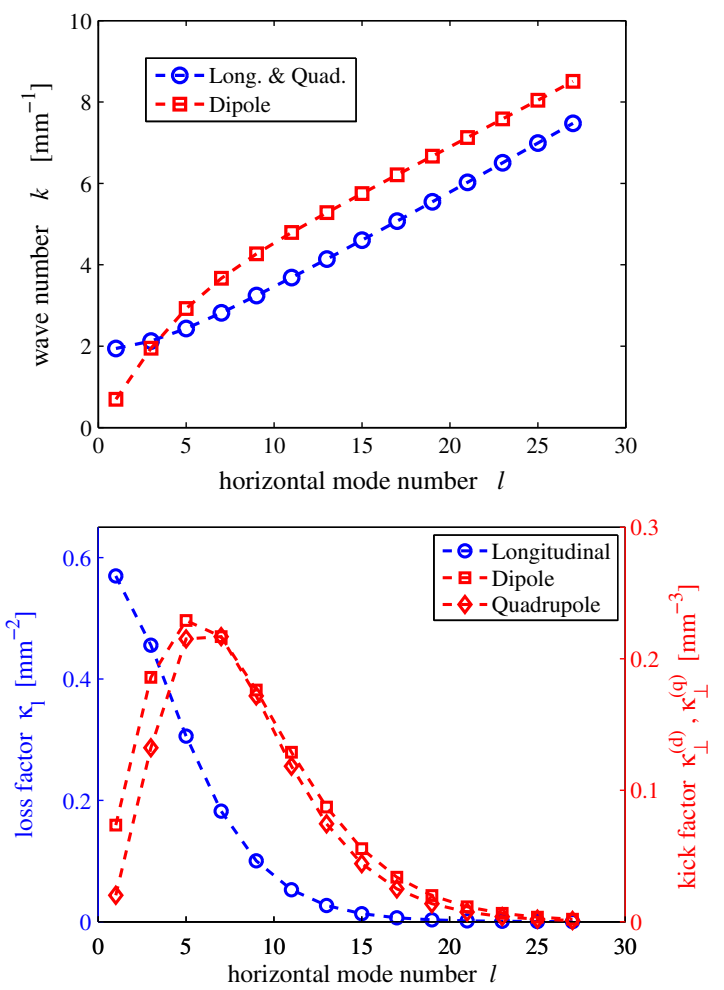

FIG. 3. (Up) wave numbers and (down) loss factor of longitudinal wake and kick factor of dipole and quadrupole wake for horizontal mode number $l$ for nominal LCLS dechirper parameters, with $a=0.7 \mathrm{~mm}$.

parameters, the contributions from $l \gtrsim 30$ can be neglected. In general, when performing these wake calculations one needs to be sure to take enough terms so that the series has converged.

\section{The wakefields}

Inserting the wave numbers, loss factors, and kick factors of Fig. 3 into Eqs. (14), (15), and (17), we obtain the longitudinal, quadrupole, and dipole wakefields, respectively, of the LCLS dechirper. The results are shown in Fig. 4 (the solid lines), where the wakes have been converted to MKS units (to convert wakes from cgs to MKS, they are simply multiplied by $Z_{0} c / 4 \pi$ ). Note that, in MKS, the units of the longitudinal and transverse wakes are $[\mathrm{MV} /(\mathrm{nCm})]$ and $\left[\mathrm{MV} /\left(\mathrm{nC} \mathrm{m}^{2}\right)\right]$, respectively. In the figure we also give the wake functions of the flat, analytical model for comparison [18] (the dashes). It is expected that the results of the field matching method agree well with the analytical model when the size of the corrugations is small compared to the gap. However, it is obvious that in the parameter regime of the LCLS dechirper, the analytical model no longer applies. The amplitudes of the wake functions with field matching are smaller than the ones of the analytical model (67\% for the longitudinal wake) and the oscillation periods also differ.
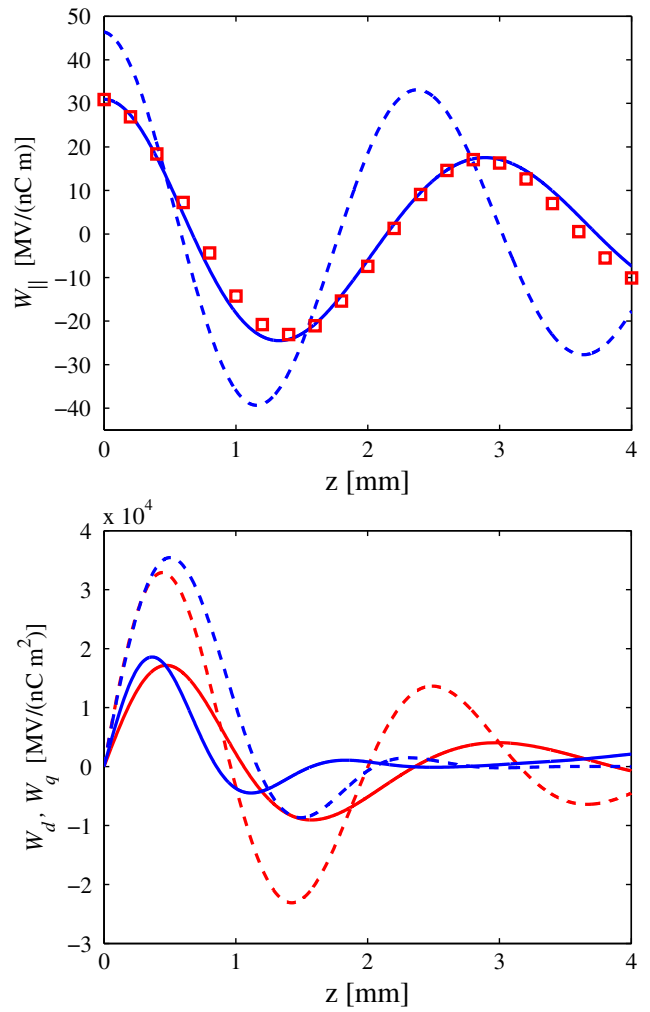

FIG. 4. Wake of the dechirper (see Table I with $a=0.7 \mathrm{~mm}$ ), as calculated using the field matching program (solid lines): (up) the longitudinal, $W_{\|}$, (down) the dipole $W_{d}$ (blue) and quadrupole $W_{q}$ (red) wake functions. The wakes according to the analytical model are also shown, for comparison (dashed lines). The fitting formula for the longitudinal wake is also shown for comparison (red square).

Based on the calculations of the field matching program, we obtain simple fitting formulas for the short-range longitudinal wakefield, which can be used for dechirper design and optimization studies (see Appendix A). The parameter range of validity is $p, h \leq a, h / p \gtrsim 0.8$, and $g / p=0.5$. The fitting formula is given by

$$
W_{\|}(z)=\frac{\pi^{2}}{16} \frac{Z_{0} c}{\pi a^{2}} F H(z) e^{-\frac{k z}{2 Q}} \cos (k z)
$$

where $F$ is an amplitude correction factor, $k$ is an effective wave number, and $Q$ is an effective quality factor. By shortrange we mean that the formula is valid for $k z \lesssim 3 \pi$. The three fitting parameters are all simple functions of structure parameters $a, h, p$. For example, the effective wave number is given by

$$
k=\frac{1}{a}\left(\frac{c_{1}}{\sqrt{h / a}}+c_{2}\right)
$$

with $c_{1}=1.7096$ and $c_{2}=-0.5026$. The expressions for $F$ and $Q$ are given in Appendix A. We show the fitting 
formula in Fig. 4 for comparison with the field matching and analytical model.

\section{Higher band modes}

Up to now, for any horizontal mode number $l$, we have considered only the lowest modes; but there are also higher such modes-i.e., higher frequency solutions to the system matrix equation-which we call "higher band modes." For a wide structure $(w / a \gg 1)$ with small corrugations, the spacing of the bands will be large compared to the spacing of the modes in the first band, and the loss factors of the modes in the higher bands will be small compared to those of the lowest modes. But when the corrugation parameters $p, h$, become comparable to $a$, the contribution of the next band modes starts to grow.

For the nominal LCLS dechirper parameters, with $a=0.7 \mathrm{~mm}$, we compare in Fig. 5 the wave numbers and loss factors of the first two band modes as functions of horizontal mode number $l$. We note that the second band mode loss factors are small, contributing about $10 \%$ of those of the first band. If we further extend the range of solving the system matrix equation, we find still higher band modes; however, in the parameter space of interest, their loss factors will be even smaller. So in this report we consider mainly the contribution of the first band modes to
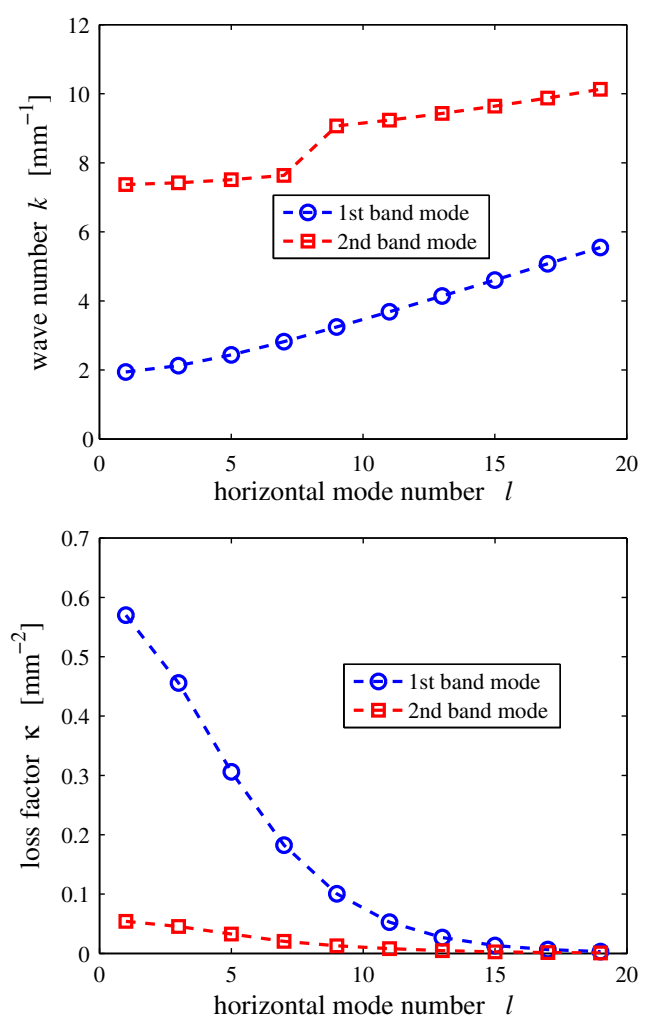

FIG. 5. Wave numbers (up) and loss factors (down) of the first two bands of modes as functions of horizontal wave number $l$, for nominal LCLS dechirper parameters with $a=0.7 \mathrm{~mm}$.

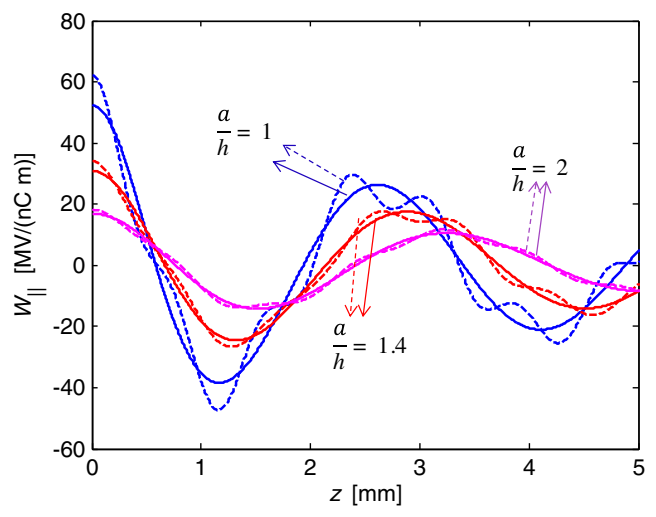

FIG. 6. Longitudinal wakefields without (solid line) and with (dashed line) the second band modes for different $a$ with other nominal parameters in Table I.

the wakes; the second band modes are calculated only for the purpose of gauging the accuracy of this approximation.

The impact of the second band modes on the wakefields is to add high-frequency features to the wakefield shape. In Fig. 6 we compare the longitudinal wakefields including only the first band modes (solid) with those including the first two bands (dashed). For the three cases considered the aperture is $a=0.5,0.7,1.0 \mathrm{~mm}$, while the other parameters are left unchanged (the $a / h=1.4$ curves correspond to the nominal parameters of Fig. 4). The distortion of the wake from a cosine shape is largest for the case of largest corrugations, i.e., for $a / h=1$. Note that such distortions have little effect on the dechirping, other than slightly changing the strength of interaction, because the bunch is very short compared to the wake oscillation period (the rms bunch length in an X-ray FEL is typically $\sigma_{z} \lesssim 20 \mu \mathrm{m}$ ).

Figure 7 gives the relative increase in the wakefield amplitude after including the second band modes as functions of $a / h$, for different $h / p$. As $h / p$ increases the effect of the second band modes first becomes larger and then settles down. For the LCLS dechirper, $h / p=1$,

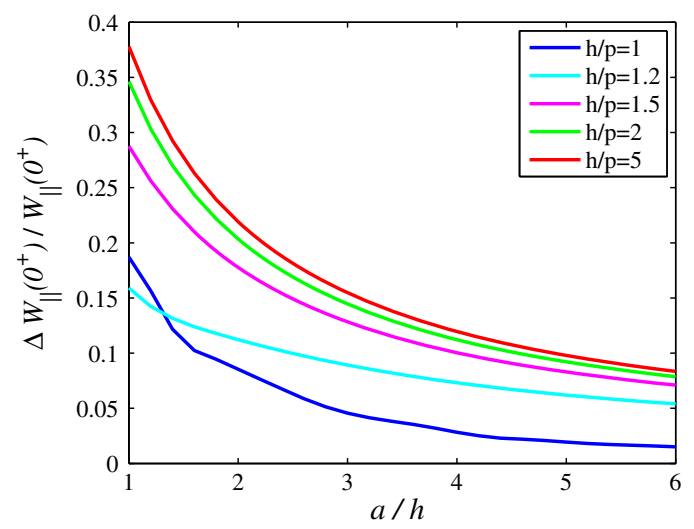

FIG. 7. Relative increase in the wakefield amplitude after including the second band modes as functions of $a / h$, for different $h / p$. Here $p, g$, have the nominal values of Table I. 


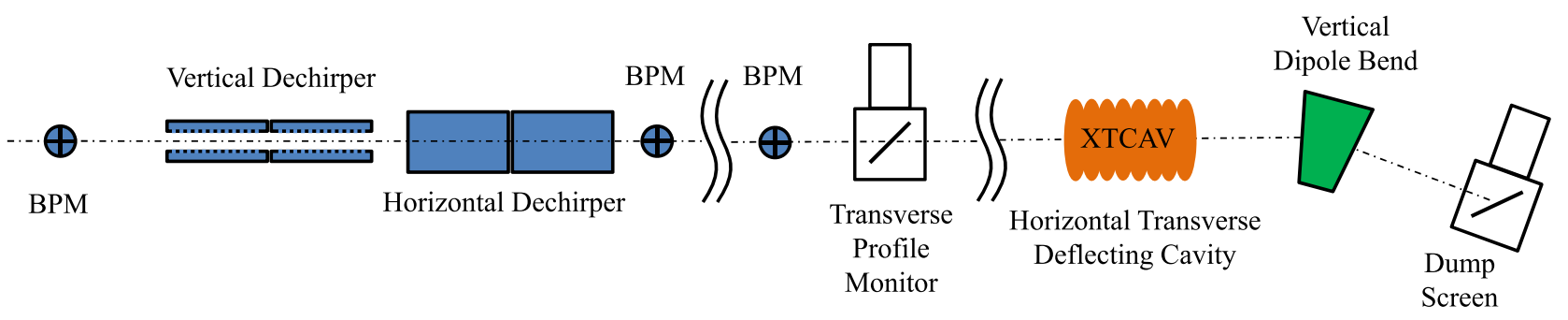

FIG. 8. Schematic of the proposed dechirper experiment (not to scale). See text for description.

so when $a \sim h=1 \mathrm{~mm}$, the amplitude when including the second band modes increases by $\sim 18 \%$. Since the fitting formula of Eq. (18) only considered the first band modes, for the LCLS dechirper, when $a$ is small $(\lesssim 1 \mathrm{~mm})$ the formula will noticeably underestimate the dechirping effect.

\section{APPLICATION TO LCLS CHIRP CONTROL}

In this section, we apply the analysis of the previous section to the proposed LCLS dechirper [13]. We also analyze other physical effects that are important.

As a demonstration experiment of chirp-control for high energy beams, the dechirper will be installed at the linac-to-undulator (LTU) area of the LCLS. Essential diagnostics for the proposed dechirper experiment are shown in Fig. 8. The dechirper will be located in a low $\beta$ region. Neighboring BPMs will be used to ensure beam alignment through the device. A distant downstream BPM will be used to detect any deflection due to the dipole wakefield. Transverse emittance measurements will be performed using LTU quads (not shown) along with the COTR-mitigated transverse profile monitor [19] at the end of LTU or, alternatively, LTU wire scanners (not shown). Finally, slice energy spread measurements will be performed in the LCLS electron dump using an X-band transverse deflecting cavity (XTCAV) [20] in conjunction with the final spectrometer bend.

The beam parameters for the proposed dechirper experiment are shown in Table II. The beam peak current after compression is $\sim 1.5 \mathrm{kA}$ and the energy chirp induced by the RF off-crest acceleration in the downstream linac is not fully canceled by the linac wakefield.

TABLE II. Beam parameters for proposed dechirper experiment.

\begin{tabular}{lcc}
\hline \hline Parameter & Value & Units \\
\hline Charge $Q$ & 150 & $\mathrm{pC}$ \\
Peak current $I_{p}$ & $\sim 1.5$ & $\mathrm{kA}$ \\
Energy $E$ & 6.6 & $\mathrm{GeV}$ \\
Emittance $\epsilon_{x}$ & 0.77 & $\mu \mathrm{m}$ \\
Emittance $\epsilon_{y}$ & 0.39 & $\mu \mathrm{m}$ \\
$\beta_{x}$ & 5 & $\mathrm{~m}$ \\
$\beta_{y}$ & 19 & $\mathrm{~m}$ \\
\hline \hline
\end{tabular}

The proposed dechirper for the LCLS is 4 meters with two 2-m sections. The total length is chosen with the aim of generating a significant dechirping effect while at the same time maintaining the beam quality. A longer dechirper will benefit the experiment as it allows a larger gap to achieve the same integrated longitudinal wake, while the transverse wakefields decrease more quickly with gap size. The parameters of the dechirper used in the simulations have been given in Table I.

The gap of the two plates can be varied in the experiment based on the required longitudinal wakefield strength. The minimum gap is $1.0 \mathrm{~mm}$ due to physical constraints and the nominal full gap size is $2 a=1.4 \mathrm{~mm}$. The longitudinal, dipole and quadrupole wakefields for this gap were presented in Fig. 4, and the beam longitudinal phase space before and after the dechirper are given in Fig. 9 by Elegant [21] simulation with beam parameters in Table II and nominal gap of the dechirper. The head of the beam lies to the left side with negative time values. The energy loss of the tail particle is $\sim 20 \mathrm{MeV}$ at nominal gap, inducing energy chirp $-0.4 \mathrm{MeV} /$ fs for 50 fs bunch length. The projected energy spread can be tuned as much as $\pm 200 \%$ of the core slice energy spread. In the experiment, we will use the XTCAV to measure the beam longitudinal phase space downstream of the FEL undulator. The final simulated images with and without the dechirper wakefields at the dump screen, which is located after the XTCAV and at a location of vertical dispersion, are given in Fig. 10. The
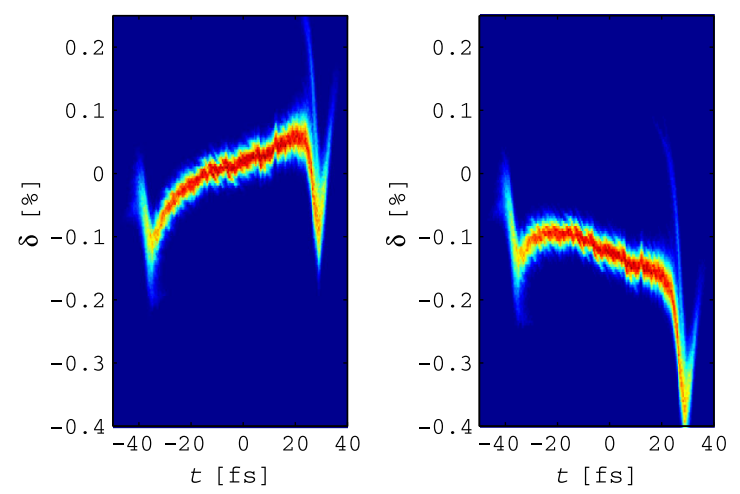

FIG. 9. Longitudinal phase space w/o (left) and w/ (right) the dechirper wakefields after the dechirper location with half-gap $2 a=1.4 \mathrm{~mm}$. Bunch head lies at the left. 

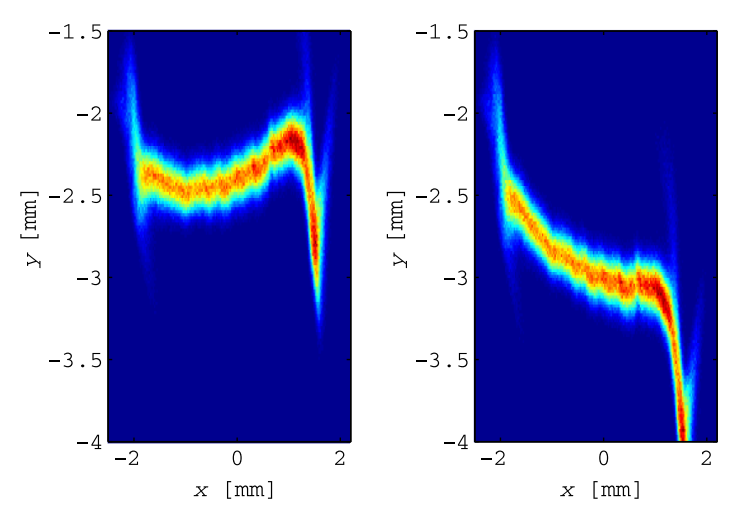

FIG. 10. Simulated images on the dump screen w/o (left) and $\mathrm{w} /$ (right) the dechirper wakefields with half-gap $2 a=1.4 \mathrm{~mm}$. Bunch head lies at the left. The simulated images appear to have deviations with longitudinal phase space in Fig. 9 due to the wakefield effects of the undulator vacuum chamber.

expected dechirping effect can be clearly measured. The simulated images at dump screen appear to have deviations with the longitudinal phase space in Fig. 9. This is due to the effects of the wakefields in the undulator vacuum chamber. During FEL operations, the gap will be varied to minimize the energy chirp of the real beam.

The dechirper can, in addition, do the opposite, i.e., it will increase the energy chirp for an overcompressed beam. The large energy chirp can help make X-ray pulses with a broader energy spectrum and generate ultrashort pulses under the self-seeding scheme [22].

\section{A. Quadrupole wakefield}

The choice of dechirper parameters is determined by the following considerations. A smaller dechirper gap generates a stronger longitudinal wakefield, and allows one to shorten the device for the required strength of effect. However, an extremely small gap makes the transverse dipole and quadrupole wakefields stronger and leads to projected emittance growth and tight tolerances on the beam position jitter and dechirper alignment. Note that the transverse wakefields used in the following tolerance study were calculated by the field matching program (see the solid lines in lower plot of Fig. 4).

The quadrupole wakefield can introduce time-dependent focusing or defocusing in the beam and increase the projected emittance. In the proposed design, the dechirper will be divided into two sections of equal length. The two sections will be oriented orthogonal to each other, one with vertical plates and the other with horizontal ones. (Here the direction of dechirper is determined by the direction of the gap, e.g., the dechirper structure shown in Fig. 1 is a vertical dechirper.) There are four possible arrangement for the two sections. We use Elegant with beam parameters in Table II and nominal gap to simulate the different combinations and compare the projected emittance in Fig. 11, which is defined as

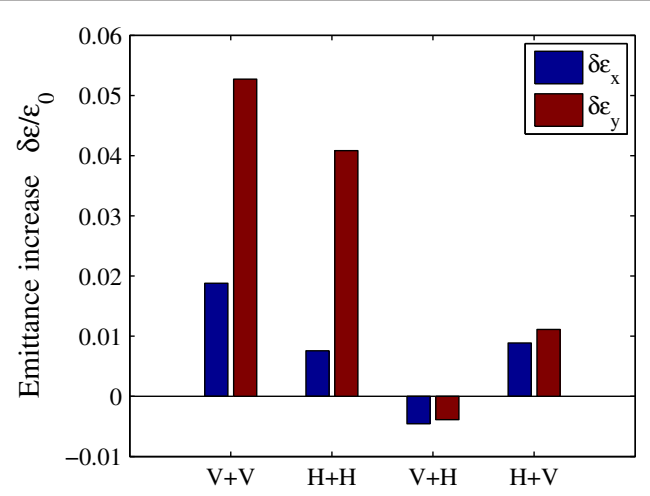

FIG. 11. Projected emittance increase for different combinations of the two dechirper sections. "V" means vertical dechirper and "H" means horizontal dechirper.

$$
\frac{\delta \epsilon}{\epsilon_{0}}=\frac{\epsilon}{\epsilon_{0}}-1
$$

where $\epsilon_{0}$ and $\epsilon$ are the emittance before and after the dechirper, respectively. The smaller increase in the horizontal plane is due to the smaller $\beta_{x}$ value and larger initial emittance $\epsilon_{x}$ before the dechirper, due to the CSR effect in upstream magnetic compression. It can be observed that if the two sections are oriented in the same direction, the projected emittance growth in the vertical plane will be $\sim 5 \%$ even when the beam and the device are both perfectly aligned. However, if the second is rotated by $90^{\circ}$ with respect to the first one, the emittance increase in both planes will become very small. That is, the quadrupole wakefield effect can be largely canceled if the two sections are crossed by $90^{\circ}$. According to Fig. 11 we adopt the "V $+\mathrm{H}$ " design for the LCLS dechirper: the first section is vertical and the second one is horizontal. It is also noticed that in the simulations in order to cancel the quadrupole wakefield we need to keep the average $\beta$ functions nearly symmetry over the structures.

We also studied the projected emittance increase versus different crossing angles of the two sections as shown in Fig. 12. When the crossing angle is not $90^{\circ}$, there will be a residual quadrupole wakefield effect that deteriorates the beam quality. However, based on the simulations, we find that the tolerance for the crossing angle is very relaxed, with $0.5 \%$ or less emittance increase even for $10^{\circ}$ deviation from the nominal.

\section{B. Dipole wakefield}

If the beam enters the structure offset from the axis, it will excite the dipole wakefield, where the tail of the beam will be kicked, increasing the projected emittance. In Fig. 13 we present the projected emittance growth versus beam offset for beam parameters in Table II with nominal gap $a=0.7 \mathrm{~mm}$. Vertical (horizontal) offset leads to vertical (horizontal) emittance growth. An analytical estimate is derived based on the method in 


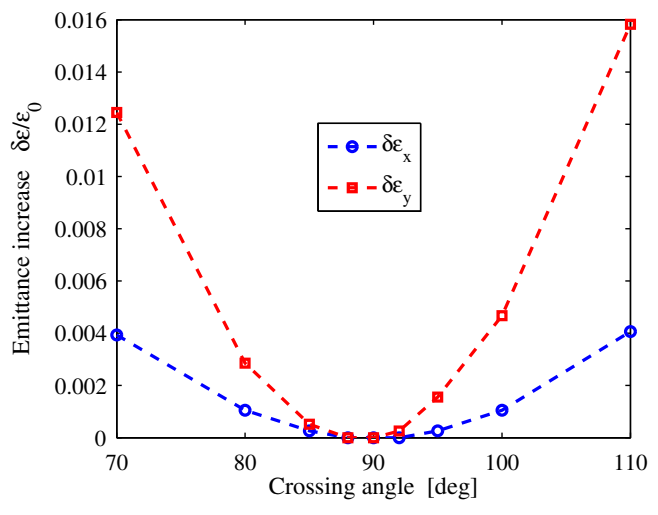

FIG. 12. Projected emittance increase versus the cross angle of the two sections.

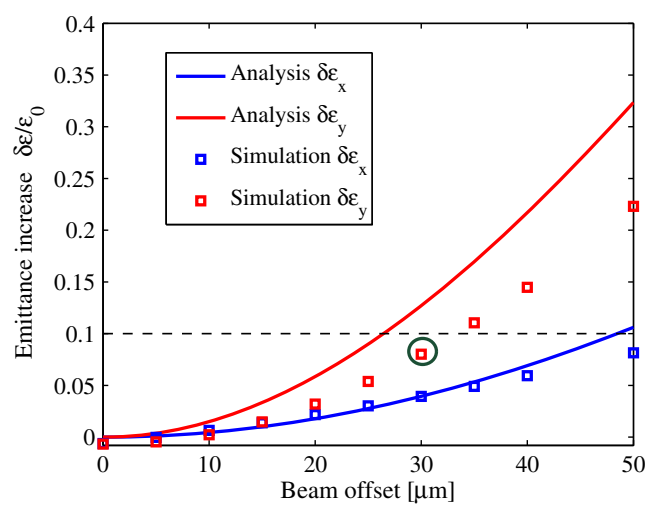

FIG. 13. Projected emittance growth versus the beam offset. Vertical (horizontal) offset leads to vertical (horizontal) emittance growth.

Ref. [8] with the fitting formulas of the longitudinal wakefields. The results are plotted in Fig. 13. We can see the emittance growth is more sensitive in the vertical plane due to the larger $\beta$ function and smaller initial emittance. In the vertical plane, the beam position jitter should be controlled to within $30 \mu \mathrm{m}$ if the allowed emittance growth is to be less than $10 \%$. This tolerance can be relaxed to $50 \mu \mathrm{m}$ for the horizontal plane. These numbers are achievable for the LCLS beam conditions and current diagnostics.

Note that this transverse jitter requirement is for the nominal gap of the dechirper. For other values, the requirement will differ. For example, if the full gap is reduced to $a=0.5 \mathrm{~mm}$ to generate larger (factor of $\sim 2$ ) longitudinal wakefield, the beam transverse position jitter should be controlled within $20 \mu \mathrm{m}$ if we want limit the emittance growth to $10 \%$. A smaller gap requires better control of the beam.

In addition to the beam offset, the alignment errors of the device will also induce dipole wakefields and increase the projected emittance, as analyzed in Appendix B. However, different from beam offset, the alignment error is steady after installation. We can offset the beam at the device entrance to find a trajectory that has no dipole kick on the beam. Thus, we are left with the requirements for transverse position jitter.

Note that the effects of the transverse wakefields also depend on the betatron functions of the beam optics. For the quadruple wakefield, it is required that the change of betatron function be small or symmetric over the device. And for dipole wakefield, smaller betatron function means looser tolerance for beam position jitter. So optimization of the optics should be performed for better performance and relaxed tolerance.

\section{CONCLUSIONS}

We have investigated the use of a pair of flat metallic plates with small corrugations as a passive device for chirp control-a "dechirper"-a type of device that will be installed in the LCLS. We studied the device's wakefields and provided numerical results obtained by field matching. When the corrugation dimensions become comparable to the gap between the two plates, the wakefields of the structure deviate from the analytical solutions that are valid for small corrugations. In addition, higher band modes arise, changing the shape of the wakefields and increasing their amplitude slightly. We also scanned the structure parameters over a large range and fit the longitudinal wake to a simple formula that can be used for quick calculation in further studies.

We then considered the application of this structure to the LCLS for the proposed chirp control, including effects of both longitudinal and transverse wakefields. Under nominal operating conditions, the 4-meter-long device will generate a large dechirping range for the LCLS beam. The energy loss of the beam tail is $\sim 20 \mathrm{MeV}$, inducing large energy chirp over short ( $\sim 50 \mathrm{fs}$ ) bunch. The projected energy spread is estimated to be as much as $\pm 200 \%$ of the core slice energy spread (with the precise amount depending on the incoming beam conditions). Through analysis and simulation, the quadrupole wake can be largely canceled by crossing the two sections of the device by $90^{\circ}$ and locally using symmetric optics. The dipole wakefield gives a tolerance on beam position jitter. If the emittance growth is to be limited to $10 \%$, the beam position jitter needs to be controlled to within $30 \mu \mathrm{m}$ for half-gap $a=0.7 \mathrm{~mm}$, and $20 \mu \mathrm{m}$ for $a=0.5 \mathrm{~mm}$. We have also studied possible structure alignment errors in the dechirper that can induce transverse wakefields and cause beam quality to deteriorate. The dipole wake due to an alignment error of the two plates can be canceled by offsetting the beam trajectory. After optimizing the beam optics in the LTU area of LCLS, mechanical stability and positioning requirements are found to be acceptable for the current beam parameters and available diagnostics. 


\section{ACKNOWLEDGMENTS}

We would like to thank P. Emma, M. Venturini, M. Ruelas, M. Harrison, P. Frigola, and D. Martin for helpful discussions. This work was supported under U.S. Department of Energy Contract No. DE-AC02-76SF00515.

\section{APPENDIX A: FITTING FORMULAS FOR THE LONGITUDINAL WAKE}

In this Appendix, we give the fitting formulas for the longitudinal wakefields. The form of the fitting formula is taken to be a damped cosine oscillation as shown as

$$
W_{\|}(z)=\frac{\pi^{2}}{16} \frac{Z_{0} c}{\pi a^{2}} F H(z) e^{-\frac{k z}{2 Q}} \cos (k z),
$$

where $F$ is an amplitude correction factor, $k$ is an effective wave number, and $Q$ is an effective quality factor. The three fitting parameters are all simple functions of structure parameters $a, h, p$. The fitting formula is valid in the specific range of the interest shown in Fig. 14. We assume the dimensions of corrugations are not larger than the gap size

$$
p, h \leq a,
$$

and the corrugation is "deeply corrugated" in order to have a strong dominant mode

$$
h / p \gtrsim 0.8 .
$$

The factor 0.8 here is chosen based on the results of numerical calculations. The applications of this structure focus on the short-range wakefields, and the longitudinal range of the fitting formulas is limited to

$$
k z \lesssim 3 \pi .
$$

Note that in the field matching calculations, we keep $g / p=1 / 2$ for simplicity.

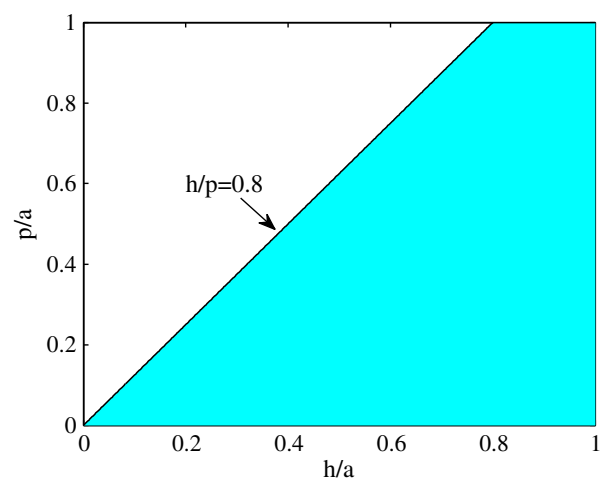

FIG. 14. Validity range (colored) of the fitting formula Eq. (18).

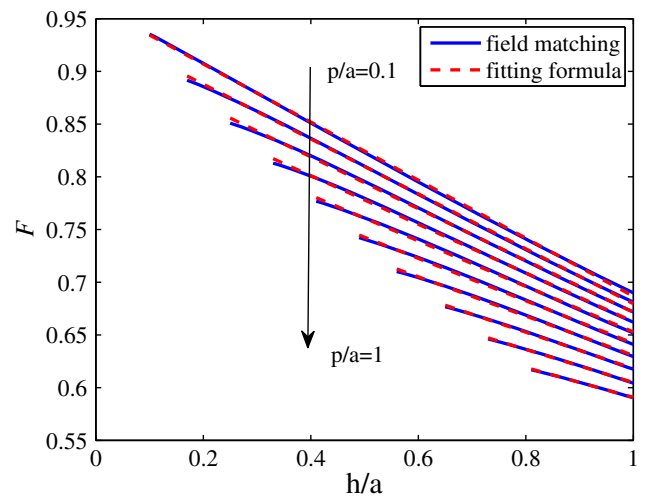

FIG. 15. Amplitude factor $F$ from field matching solution (blue solid line) and corresponding best fit to Eq. (A5) (red dashed line).

The field matching and the fitting results for $F, k$, and $Q$ are shown in Figs. 15, 16, and 17. Each line in Fig. 15 corresponds to a value of $p / a$ from 0.1 to 1 and the range of $h / a$ is determined by the requirement Eq. (A3). The double-arrow line in Figs. 16 and 17 signify that for a given $h / a$, we plot all wave numbers under different $p / a$ values ranging from 0.1 to 1 . Based on these results, we can obtain the formulas for the three fitting parameters. The fitting forms for the parameters:

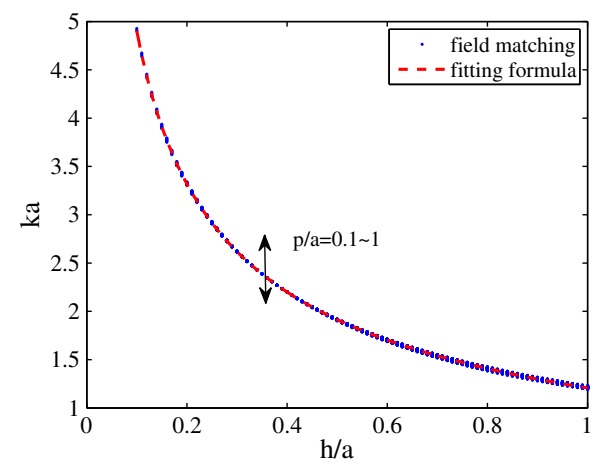

FIG. 16. Wave number by field matching algorithm (blue dots) and corresponding best fit to Eq. (A6) (red dashed line).

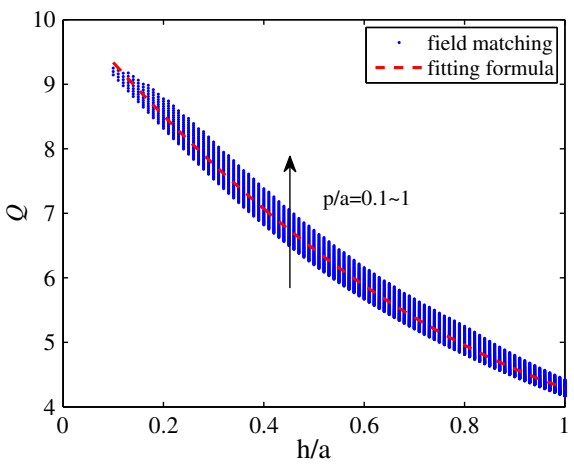

FIG. 17. Quality factor by field matching algorithm (blue dots) and corresponding best fit to Eq. (A7) (red dashed line). 
TABLE III. Coefficients of best fit for Eqs. (A5), (A6), (A7) to the field matching calculations.

\begin{tabular}{lccc}
\hline \hline Coefficient & Value & Coefficient & Value \\
\hline$b_{1}$ & 0.1483 & $c_{1}$ & 1.7096 \\
$b_{2}$ & 0.1418 & $c_{2}$ & -0.5026 \\
$b_{3}$ & -0.0437 & $d_{1}$ & 3.2495 \\
$b_{4}$ & 0.1460 & $d_{2}$ & -9.1830 \\
$b_{5}$ & 0.5908 & $d_{3}$ & 10.2230 \\
\hline \hline
\end{tabular}

$$
\begin{aligned}
F\left(\frac{h}{a}, \frac{p}{a}\right)= & b_{1}\left(1-\frac{p}{a}\right)\left(1-\frac{h}{a}\right)+b_{2}\left(1-\frac{h}{a}\right) \\
+ & b_{3}\left(1-\frac{p}{a}\right)^{2}+b_{4}\left(1-\frac{p}{a}\right)+b_{5}, \\
k & =\frac{1}{a}\left(\frac{c_{1}}{\sqrt{h / a}}+c_{2}\right), \\
Q\left(\frac{h}{a}\right) & =d_{1}\left(\frac{h}{a}\right)^{2}+d_{2} \frac{h}{a}+d_{3},
\end{aligned}
$$

where $b_{i}(i=1, \ldots, 5), c_{1}, c_{2}, d_{1}, d_{2}, d_{3}$ are the fitting coefficients. The best fit result for these coefficients is given in Table III.

The results of the fitting formulas are also indicated in the corresponding figures by red dashed lines. It can be seen that for the amplitude factor $F$ and wave number $k$ the fitting formulas agree well with the results of the field matching program. The sum of the five coefficients $F(0,0)=\sum_{i=1}^{5} b_{i} \approx 1$ indicates that the new formula is consistent with the analytical formula as $p / a \ll 1$, $h / a \ll 1$. For the wave number $k$ and quality factor $Q$, the precision is also good enough for applications which focus on the short-range wakefields, such as for energy chirp control.

\section{APPENDIX B: DECHIRPER STRUCTURE ERRORS}

We study here the effect of the structure rotation error as shown in Fig. 18. The two planes of the dechirper can both have a rotation angle with respect to the beam direction, which can induce dipole wakefields, even when the beam passes through the structure on axis.

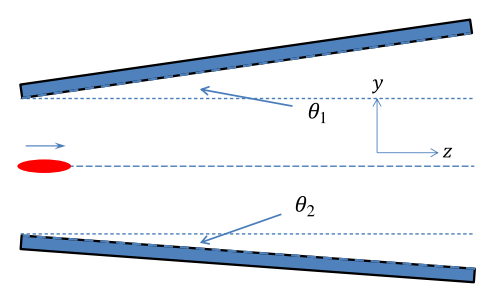

FIG. 18. Dechirper with x-rotation error. Anticlockwise (clockwise) rotation corresponds to positive (negative) angle.
The period of the corrugation is much shorter than the length of the dechirper and the rotation angles (denoted by $\theta_{1}, \theta_{2}$ in Fig. 18) should be small, so we can assume that within a short longitudinal range, the corrugations can be viewed as of a constant gap size. Then we can write down the emittance growth due to the dipole wakefield within a small rang $s \rightarrow s+\Delta s$

$$
\begin{aligned}
& {\left[\sqrt{\left(\frac{\epsilon}{\epsilon_{0}}\right)^{2}-1}\right]_{s \rightarrow s+\Delta s}} \\
& =0.375 Z_{0} c \cdot \frac{F q \beta \sigma_{z} \Delta s}{\left[a+s\left(\theta_{1}-\theta_{2}\right) / 2\right]^{4} E} \cdot \frac{s\left(\theta_{1}+\theta_{2}\right)}{2 \sigma_{0}},
\end{aligned}
$$

with $q$ bunch charge, $\sigma_{z}$ bunch length, $\sigma_{0}$ transverse beam size, $E$ beam energy, $\beta$ the twiss parameter and $F$ the amplitude factor of dipole wakefield calculated by the field matching. For simplicity, we choose $\theta_{1}$ as the variable and set $\theta_{2}=0$, then we can integrate Eq. (B1) over the beam path in the 2-meter-long section to get the effect of the dipole wakefield. The projected emittance increase versus the rotation angle $\theta_{1}$ is given in Fig. 19. The vertical projected emittance will increase by $\sim 9 \%$ if one plane of the dechirper is tilted $0.1 \mathrm{mrad}$ $\left(\theta_{1}=0.1 \mathrm{mrad}, \theta_{2}=0\right)$.

However, for a given rotation angle we can cancel the dipole wakefield effect by offsetting the beam at the entrance. Assuming the beam is offset by $\Delta$, Eq. (B1) can be rewritten as

$$
\begin{aligned}
& {\left[\sqrt{\left(\frac{\epsilon}{\epsilon_{0}}\right)^{2}-1}\right]_{s \rightarrow s+\Delta s}} \\
& =0.375 Z_{0} c \cdot \frac{F q \beta \sigma_{z} \Delta s}{\left[a+s\left(\theta_{1}-\theta_{2}\right) / 2\right]^{4} E} \cdot \frac{s\left(\theta_{1}+\theta_{2}\right) / 2-\Delta}{\sigma_{0}},
\end{aligned}
$$

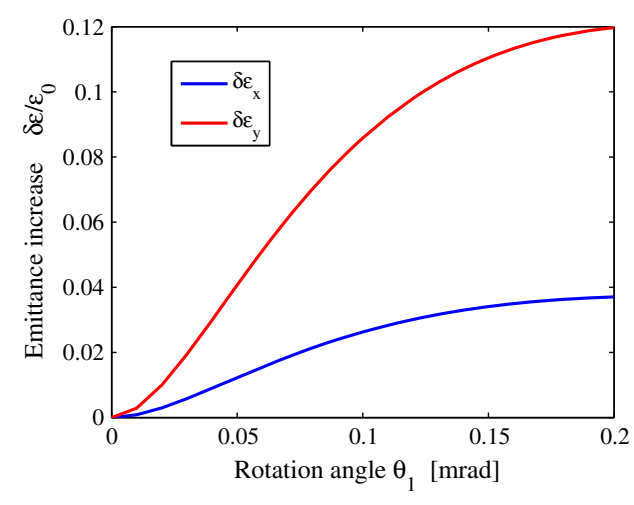

FIG. 19. Emittance increase versus the rotation angle $\theta_{1}$ $\left(\theta_{2}=0\right)$ for a 2 -meter-long section. 


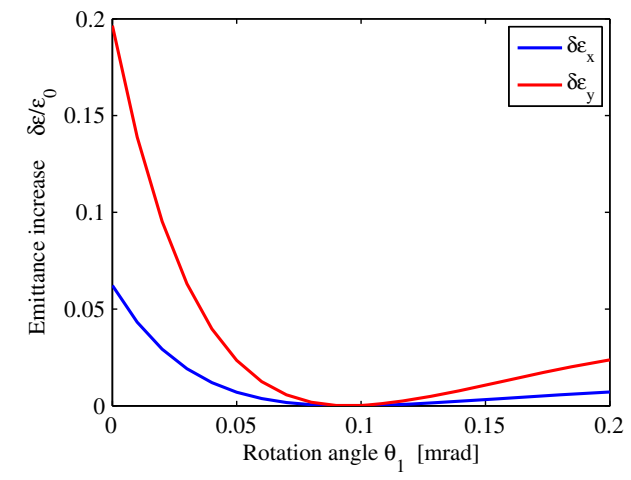

FIG. 20. Emittance increase versus the rotation angle $\theta_{1}$ $\left(\theta_{2}=0\right)$ for a 2-meter-long section with beam offset $\Delta=40 \mu \mathrm{m}$.

For example, if the rotation angles of the two planes are $\theta_{1}=0.1 \mathrm{mrad}$ and $\theta_{2}=0$, we can offset the beam in $y$ in Fig. 18 by $+40 \mu \mathrm{m}$ to cancel the dipole wakefield, which can be seen in Fig. 20. Although the analysis here is only for the angle errors of the two plates, it can be also applied to other structure errors that can excite the dipole wakefields.

[1] W. Ackerman et al., Nat. Photonics 1, 336 (2007).

[2] P. Emma et al., Nat. Photonics 4, 641 (2010).

[3] H. Tanaka et al., Nat. Photonics 6, 540 (2012).

[4] E. Allaria et al., Nat. Photonics 6, 699 (2012).

[5] S. Krinsky and Z. Huang, Phys. Rev. ST Accel. Beams 6, 050702 (2003).

[6] E. Saldin, E. Schneidmiller, and M. Yurkov, Phys. Rev. ST Accel. Beams 9, 050702 (2006).
[7] K. Bane and G. Stupakov, Nucl. Instrum. Methods Phys. Res., Sect. A 690, 106 (2012).

[8] K. Bane and G. Stupakov, Report No. SLAC-PUB-15852, 2013 and Report No. LCLS-II TN-13-01.

[9] P. Emma et al., Phys. Rev. Lett. 112, 034801 (2014).

[10] M. Harrison et al., in Proceedings of the 25th Particle Accelerator Conference, PAC-2013, Pasadena, CA, 2013 (IEEE, New York, 2013).

[11] H.X. Deng et al., Phys. Rev. Lett. 113, 254802 (2014).

[12] K. Bane et al., Report No. SLAC-PUB-15853, 2013 and Report No. LCLS-II TN 13-02.

[13] M. Ruelas et al., in 36th International Free Electron Laser Conference, FEL2014, Basel, Switzerland, 2014, THP033, http://www.fel2014.ch/prepress/FEL2014/papers/thp033 .pdf.

[14] G. Stupakov and K. Bane, Phys. Rev. ST Accel. Beams 15, 124401 (2012).

[15] K. Bane and G. Stupakov, Phys. Rev. ST Accel. Beams 6, 024401 (2003).

[16] K. Bane and A. Novokhatski, SLAC Report No. LCLSTN-99-1, 1999.

[17] K. Bane and G. Stupakov, in Proceedings of the 20th International Linac Conference, LINAC-2000, Monterey, CA, 2000 (SLAC, Menlo Park, CA, 2000), p. 92.

[18] G. Stupakov (unpublished).

[19] R. Ischebek et al., in Proceedings of the 2nd International Beam Instrumentation Conference, Oxford, UK, 2013, http://accelconf.web.cern.ch/AccelConf/IBIC2013/papers/ mobl1.pdf.

[20] C. Behrens et al., Nat. Commun. 5, 3762 (2014).

[21] M. Borland, ELEGANT, Advanced Photon Source LS-287, 2000.

[22] C. B. Schroeder et al., J. Opt. Soc. Am. B19, 1782 (2002). 